\title{
Association of Physical Activity and Derived Neutrophil- to-Lymphocyte Ratio with Outcome in Patients with Recurrent and/ or Metastatic Squamous Cell Carcinoma of Head and Neck Treated with Immunotherapy
}

\author{
Pesantez $\mathrm{D}^{1 *}$, Basté $\mathbf{N}^{1}$, Oberoi HK${ }^{1}$, Castillo $\mathbf{P}^{2}$, \\ Berenguer $\mathrm{J}^{3}$, Vilaseca $\mathrm{I}^{4,5}$ and Grau JJ ${ }^{1,5}$ \\ ${ }^{1}$ Department of Medical Oncology, Hospital Clinic of \\ Barcelona, Spain \\ 2Department of Pathology, Hospital Clinic of Barcelona, \\ Spain \\ ${ }^{3}$ Department of Neuroradiology, Hospital Clinic of \\ Barcelona, Spain \\ ${ }^{4}$ Department of Otolaryngology, Hospital Clinic of \\ Barcelona, Spain \\ ${ }^{5}$ University of Barcelona, Agusti Pi Sunyer Biomedical \\ Research Institute (IDIBAPS), Spain \\ *Corresponding author: David Pesántez, Department \\ of Medical Oncology, Hospital Clínic de Barcelona, C/ \\ Villarroel, 170, 08036, Barcelona, Barcelona, Spain
}

Received: January 25, 2021; Accepted: February 27, 2021; Published: March 06, 2021

\begin{abstract}
Regular Physical Activity (PA) improves the outcomes of patients with cancer mainly by enhancing the immune system. The relationship of PA and the derived Neutrophil-to-Lymphocyte Ratio (dNLR) with the evolution of 31 consecutive patients with Recurrent and/or Metastatic Squamous Cell Carcinoma of Head and Neck (R/M SCCHN) treated with immunotherapy was determined in this retrospective study.

Seventeen patients (55\%) performed PA and 14 (45\%) did not. The time to progression and Overall Survival (OS) was significantly better in the first compared to the second group ( $\mathrm{p}=0.002$ and 0.0019 , respectively). In patients with a dNLR less than 3.5 the survival was significantly longer than in patients with a higher $\mathrm{dNLR}(\mathrm{p}=0.004)$.
\end{abstract}

Our results suggest that there is an association between PA and improved outcomes in R/M SCCHN patients treated with immunotherapy and that the $\mathrm{dNLR}$ is a predictive marker of good response to treatment.

Keywords: Head and neck cancer; Physical activity; Immunotherapy; Derived neu-trophil-to-Lymphocyte ratio

\section{Introduction}

Head and neck cancer is a significant global health problem, accounting for over 650,000 cases and 330,000 deaths annually [1]. Patients are usually diagnosed with locoregional disease and the mainstream treatment is surgery and/or chemoradiotherapy. Despite receiving aggressive treatment, more than $50 \%$ of patients relapse with inoperable locally advanced or distant disease. Since 2008, standard treatment in patients with R/M SCCHN has been chemotherapy based on a platinum compound combined with 5 -flurouracil and cetuximab [2]. Recently, nivolumab and pembrolizumab have proven to have benefits in terms of survival in patients with platinum refractory diseases $[3,4]$. Durvalumab and durvalumab + tremelimumab have also shown clinical benefits in patients with R/M SCCHN and low or no PD-L1 tumour cell expression, with few differences being observed between the two. A phase 3 study on the use of these drugs is currently ongoing [5].

It has been suggested that regular PA following the diagnosis of certain solid tumours may improve treatment outcomes, reducing disease progression and mortality [6]. In-deed, several studies have suggested that regular PA can modify the tumoral microenvironment by changing the distribution of immune cells, thereby achieving better anti-tumor response [7].

Mice models of SCCHN have shown a reduced number and activity of natural killer cells following inhibition with TGF-beta-1 [8]. Additionally, lower lymphocyte counts have been found in patients with SCCHN compared to healthy controls [9], and it has been proposed that a peripheral proinflammatory state is associated with poor response in cancer patients $[10,11]$. In this context, the utility of the derived neutrophil-to-lymphocyte ratio (dNLR) has been explored and confirmed as a prognostic and predictive factor in relation to immunotherapy, mostly in melanoma and non-small cell lung cancer [12-14], and this biomarker has become widely available in routine clinical practice because it is inexpensive and easy to obtain.

We hypothesised that daily PA, defined as aerobic walking outdoors for 1 hour a day, could modify the balance between the innate and adaptive immune system, towards the latter, increasing response to immunotherapy in patients with R/M SCCHN. However, many patients refuse to go outside because of facial cosmetic problems or discomfort due to tracheostomy and so, are not benefiting from the possible positive effect of PA and immunotherapy treatment [3]. Therefore, the objective of this study was to establish a potential correlation between clinical and biological factors (such as PA and the dNLR) and response to immunotherapy, time-to-progression and OS in patients with R/M SCCHN.

\section{Methods}

\section{Patients}

The inclusion criteria were pathologically confirmed $\mathrm{R} / \mathrm{M}$ SCCHN of the oral cavity, pharynx or larynx not amenable to curative treatment; having received anti-PD-1 or anti-PD-L1 either in routine practice or within a clinical trial; having a performance-status score of 
$\leq 2$; and the absence of physical or mental limitations to perform the minimum PA required.

\section{Treatment and assessments}

Nivolumab was administered at a dose of $240 \mathrm{mg}$ every 2 weeks until disease progression or unacceptable toxicity. Other anti-PD-L1 or anti-PD1 drugs (durvalumab, $10 \mathrm{mg} / \mathrm{k}$ or avelumab, $10 \mathrm{mg} / \mathrm{k}$ ) were administered according to a clinical trial protocol until disease progression or unacceptable toxicity. All patients were invited to perform daily PA consisting in walking outdoors for at least 1 hour/ day prior to initiation and during treatment with immunotherapy. Compliance with PA was registered in the medical records for further analysis. According to what the patients referred, they were assigned to Group A (correctly carried at least a $75 \%$ out the recommended PA) or Group B (they carried less than a $75 \%$ of the recommended PA). No quantitative method to define the PA was used.

All patients underwent medical examination and a complete blood test, including cell counts and serum chemistry, before each treatment infusion as a part of routine clinical practice.

The dNLR was defined as an Absolute Neutrophil Count (ANC)/ (White Blood Cells (WBC) - ANC) [10]. The dNLR was determined in all patients regardless of their PA. Tumour assessment was carried out at baseline, at week 12 and every 12 weeks thereafter, and clinical response was classified according to immune-related response criteria (iRecist) [15].

OS was defined as the time from treatment initiation to death (event) or last control of the patient. Progression-Free Survival (PFS) was defined as the time from treatment initiation to disease progression or death whichever occurred first.

\section{Statistical analysis}

Analyses of efficacy followed the intention-to-treat principle. We used descriptive statistics to define patient characteristics and used parametric and nonparametric tests to establish differences. Based on previous retrospective studies, the cut-off point for the dNLR was established at 3.5. For the univariate analysis of OS and diseasefree survival, and response duration or PFS, we used Kaplan-Meier survival curves compared with the log rank test.

\section{Results}

Thirty-one consecutive patients treated with Immune-Checkpoint Inhibitors (ICI) were included between May 2016 and July 2017. Treatment consisted in the administration of nivolumab $(\mathrm{n}=21)$, durvalumab $(n=9)$ or avelumab $(n=1)$. The basal characteristics of the patients studied are shown in (Table 1).

Fourteen of the 31 patients included (45\%) carried out at least $75 \%$ of the recommended PA (Group A) while 17 out of 31 patients (55\%) carried out less than $75 \%$ of the recommended PA (Group B). The reasons for not performing the PA included discomfort or problems related to social contact due to tracheotomy or an alteration in facial esthetics. Four patients (13\%) showed objective response and 5 (16\%) stable disease. Nine of 31 patients (29\%), obtained clinical benefit. Seven (50\%) from Group A and 2 (12\%) from Group B.

The median follow-up was 9 months with a median duration of response of 8 months (95\% Confidence Interval [CI]: 3.120-12.880).

\section{Time to progression (months)}

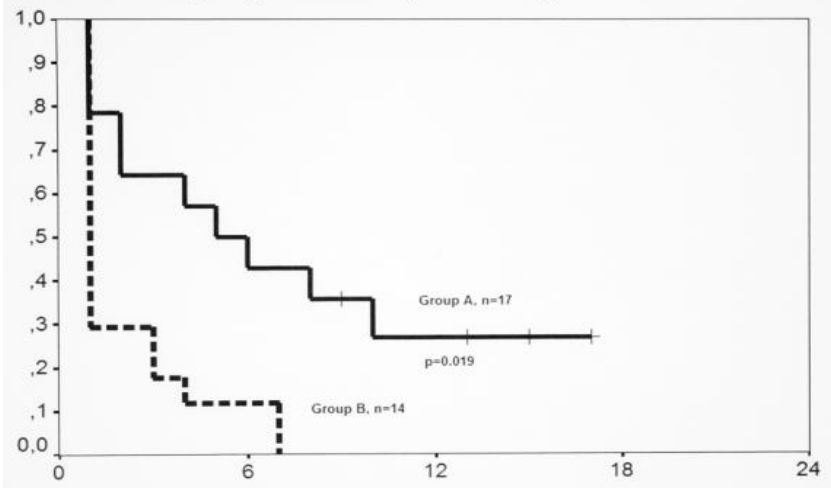

Figure 1: Time to progression in patients who performed physical activity (Group A, $n=17$ ) versus those who did not (Group B, $n=14)(p=0.002)$.

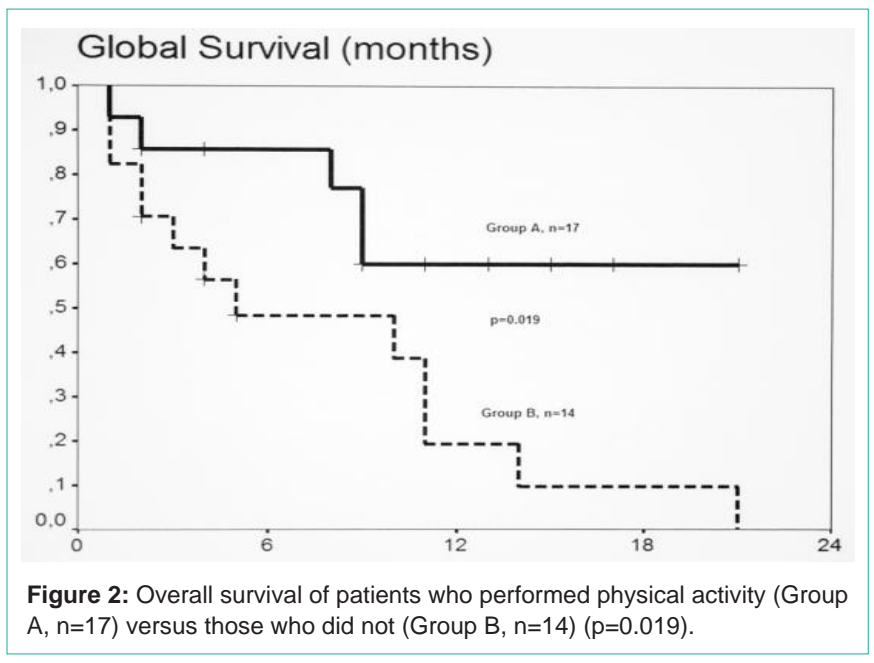

The median PFS was significantly better in Group A versus Group B (5 versus 1 month, respectively, $\mathrm{p}=0.002$ ) (Figure 1). The median OS was also better in Group A compared to Group B (9 versus 4 months, $\mathrm{p}=0.019$ ) (Figure 2).

\section{dNLR in blood test}

A low baseline dNLR (less than 3.5) showed statistically differences in the median OS versus those patients with dNLR equal or over 3.5 (21 versus 4 months, $\mathrm{p}=0.004$ ) (Figure 3 ). The expression of dNLR following the first dose of immunotherapy (dNLRpost) was only determined in 28 patients since 3 did not receive the second treatment cycle. In 7 of these 28 patients (25\%) the dNLR status changed from high to low, and these patients belonged to group A. According to the dNLRpost, the OS was significantly in favour of patients with a value lower than the established cut-off ( $\mathrm{p}=0.007)$.

\section{PD-L1 tumour expression}

Biopsies of the primary tumour were retrospectively analyzed to determine PD-L1 expression, and in 5 patients PD-L1 could not be determined due to lack of or poor preservation of material. Of the 26 patients analyzed, the Total Positive Score (TPS) was greater than or equal to $50 \%$ in 2 patients: one from Group A and the other from Group B ( $p=0.328)$. The Combined Positive Score (CPS) was greater 


\section{Survival According dNLR}

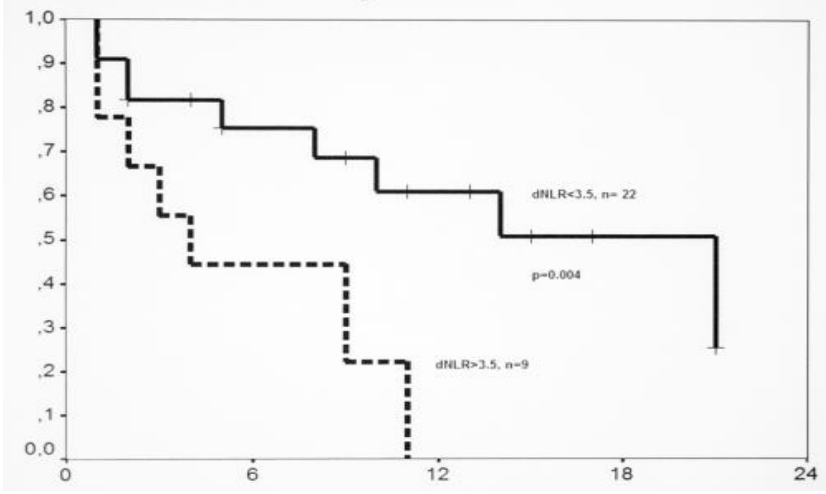

Figure 3: Overall survival according to the dNLR at baseline (cut-off 3.5). The over-all survival was significantly better in patients with nNLR expression less than $3.5(p=0.004)$

Table 1: Baseline characteristics of the patients studied.

\begin{tabular}{|c|c|c|c|}
\hline & ALL & GROUP A & GROUP B \\
\hline $\mathrm{n} / \%$ & $31(100 \%)$ & $14(45 \%)$ & $17(55 \%)$ \\
\hline \multicolumn{4}{|l|}{ Gender } \\
\hline Male & $24(77 \%)$ & $11(78 \%)$ & $13(76 \%)$ \\
\hline Female & $7(23 \%)$ & $3(22 \%)$ & $4(24 \%)$ \\
\hline AGE (median) & $61(49-85)$ & $57(49-75)$ & $63(54-85)$ \\
\hline \multicolumn{4}{|l|}{ ECOG } \\
\hline 1 & $30(97 \%)$ & $14(100 \%)$ & $16(94 \%)$ \\
\hline 2 & $1(3 \%)$ & $0(0 \%)$ & $1(6 \%)$ \\
\hline \multicolumn{4}{|l|}{ Primary Tumour } \\
\hline Rhinosinusal & $2(6 \%)$ & $1(7 \%)$ & $1(6 \%)$ \\
\hline Oropharynx & $15(48 \%)$ & $5(36 \%)$ & $10(59 \%)$ \\
\hline Hypopharynx & $12(40 \%)$ & $7(50 \%)$ & $5(29 \%)$ \\
\hline Larynx & $2(6 \%)$ & $1(7 \%)$ & $1(6 \%)$ \\
\hline HPVp16 + & $3(10 \%)$ & $1(7 \%)$ & $2(12 \%)$ \\
\hline \multicolumn{4}{|l|}{ Previous Treatment } \\
\hline Chemoradiotherapy & $8(26 \%)$ & $4(29 \%)$ & $4(24 \%)$ \\
\hline Platinum based (Extreme) & $23(74 \%)$ & $10(71 \%)$ & $13(76 \%)$ \\
\hline TPS $>50$ & $2(100 \%)$ & $1(7 \%)$ & $1(6 \%)$ \\
\hline CPS $>1$ & $13(100 \%)$ & $5(35 \%)$ & $8(35 \%)$ \\
\hline CPS $>20$ & $2(100 \%)$ & $1(7 \%)$ & $1(6 \%)$ \\
\hline
\end{tabular}

TPS: Total Positive Score; CPS: Combined Positive Score.

than or equal to 1 in 13 patients ( 5 from Group A and 8 from Group B) ( $p=0.656)$. A CPS $\geq 20$ was observed in 2 patients, one from Group $A$ and the other from Group B ( $p=0.348)$. No significant differences were observed in the rate of response, PFS or OS according to PD-L1 status.

\section{Discussion}

According to our hypothesis, the results of the present study suggest that there is an association between regular PA and improved outcomes in patients with $\mathrm{R} / \mathrm{M}$ SCCHN receiving immunotherapy. To our knowledge, there is no previous report in this regard.
Numerous studies have related immune response with PA in patients with cancer $[16,17]$. One major potential anticancer effect of exercise is related to enhancement of immune function, with moderate PA stimulating the innate immune system, especially natural killer cells [16-18]. It has also been suggested that PA can positively modify the NLR [19]. This relationship has been reported as a prognostic factor in patients with colorectal cancer [20,21], and it has also been studied as a predictive factor of response and good evolution in patients with lung cancer treated with immunotherapy [12].

It is, therefore, reasonable to hypothesize that active PA could improve response to anti-PD-1/PD-L1 agents in the treatment of cancer patients by better distribution of cytotox-ic $\mathrm{T}$ lymphocytes $[22,23]$. The dNLR is easy to calculate with data obtained in routine blood tests prior to treatment with immunotherapy and could potentially be used as a prognostic factor. There is no standard cut-off point for use in all types of cancer, but most studies use a ratio between 3 and 4 [24].

On the other hand, the analysis of PD-L1 tumour expression in our cohort did not show any differences in the response rate or response duration, probably due to the small number of patients analyzed. The main limitation of the present study is the small size of the cohort and the type of PA registry, which do not allow definitive conclusions to be made. PA is difficult to measure for the following reasons: 1) there are at least four domains: occupational, household, transportation and leisure time; 2) PA questionnaires are subject to recall bias; and 3) objective methods can only be used in prospective studies for short time periods [16,27].

In conclusion, the results of this retrospective study in patients with R/M SCCHN treated with immunotherapy suggest that regular PA improves the evolution of patients and that the dNLR, which is easy to perform in daily clinical practice, could be a predictive marker of good response to treatment. Nonetheless, despite the increasing number of studies addressing the benefits of exercise in cancer patients, further controlled trials are needed to clarify the optimal intensity and duration of PA and many unanswered questions such as which patients can be benefited with this approach.

\section{Acknowledgements}

We would like to thank BMS Spain for their financial support in the retrospective analysis of PD-L1 in the primary tumours of the patients studied.

\section{Declarations}

Funding: BMS Spain contributed to the funding of the retrospective analyses of the $\mathrm{PD}-\mathrm{L} 1$ expression in the tumor.

\section{Contributions}

All authors were involved in the conception of this work, drafting and/or revising the manuscript, and approved the final version.

Ethical approval: The manuscript does not include any prospective study with human participants or animals.

Informed consent: All the patients studied provided informed consent/ethical approval to publish patient data. 


\section{References}

1. Global cancer statistics 2018: GLOBOCAN estimates of incidence and mortality worldwide for 36 cancers in 185 countries. CA Cancer J Clin. 2018 68: $394-424$

2. Vermorken JB, Mesia R, Rivera F, Remenar E, Kawecki A, Rottey S, et al Platinum-base chemotherapy plus cetuximab in head and neck cancer. $N$ Engl J Med. 2008; 359: 1116-1127.

3. Ferris RL, Blumenschein G, Fayette J, Guigay J, Colevas AD, Licitra L, et al Nivolumab for recurrent squamous-cell carcinoma of the head and neck. $N$ Engl J Med. 2016; 375: 1856-1867.

4. Cohen EEW, Soulières $D$, Le Tourneau C, Dinis J, Licitra L, Ahn MJ, et al. KEYNOTE-040 investigators. Pembrolizumab versus methotrexate docetaxel, or cetuximab for recurrent or metastatic head-and-neck squamous cell carcinoma (KEYNOTE-040): a randomised, open-label, phase 3 study. Lancet. 2019; 393: 156-167.

5. Siu LL, Even C, Mesia R, Remenar E, Daste A, Delord JP, et al. Safety and Efficacy of Durvalumab With or Without Tremelimumab in Patients With PDL1-Low/Negative Recurrent or Metastatic HNSCC: The Phase 2 CONDOR Randomized Clinical Trial. JAMA Oncol. 2019; 5: 195-203.

6. Friedenreich CM, Neilson HK, Farris MS, Courneya KS. Physical activity and cancer outcomes: a precision medicine approach. Clin. Cancer Res. 2016 22: $4766-4775$

7. Koelwyn GJ, Quail DF, Zhang X, White RM, Jones LW. Exercise-dependen regulation of the tumour microenvironment. Nat Rev Cancer. 2017; 17: 620632.

8. Dasgupta S, Bhattacharya-Chatterjee M, O'Malley BW, Chatterjee SK. Inhibition of NK cell activity through TGF-beta 1 by down-regulation of NKG2D in a murine model of head and neck cancer. J Immunol. 2005; 175 5541-5550.

9. Kuss I, Hathaway B, Ferris RL, Gooding W, Whiteside TL. Decreased absolute counts of $\mathrm{T}$ lymphocyte subsets and their relation to disease in squamous cell carcinoma of the head and neck. Clin Cancer Res off J Am Assoc Cancer Res. 2004; 10: 3755-3762.

10. Proctor MJ, McMillan DC, Morrison DS, et al. A derived neutrophil to lymphocyte ratio predicts survival in patients with cancer. Br J Cancer. 2012 107: 695-699.

11. McMillan DC. The systemic inflammation-based Glasgow Prognostic Score: a decade of experience in patients with cancer. Cancer Treat Rev. 2013; 39: $534-540$.

12. PF Ferrucci, PA Ascierto, J Pigozzo, M Del Vecchio, M Maio, GC Antonin Cappellini, et al. Baseline neutrophils and derived neutrophil-to-lymphocyte ratio: prognostic relevance in metastatic melanoma patients receiving ipilimumab. Annals of Oncology. 2016; 27: 732-738.

13. Mezquita L, Auclin E, Ferrara R. Association of the Lung Immune Prognostic Index with Immune Checkpoint Inhibitor Outcomes in Patients with Advanced Non-Small Cell Lung Cancer. JAMA Oncol. 2018; 4: 351-357.
14. Cedrés S, Torrejon D, Martínez A. Neutrophil to Lymphocyte Ratio (NLR) as an indicator of poor prognosis in stage IV non-small cell lung cancer. Clin Transl Oncol. 2012; 14: 864-869.

15. Wolchok JD, Hoos A, O'Day S. Guidelines for the evaluation of immunetherapy activity in solid tumors: immune-related response criteria. Clin Cancer Res. 2009; 15: 7412-7420.

16. Pollán M, Casla-Barrio S, Alfaro J. Exercise and cancer: a position statement from the Spanish Society of Medical Oncology. Clin Transl Oncol. 2020; 22: 1710-1729.

17. Figueira A, Cortinhas A, Soares J, Leitão J, Ferreira R, Duarte J. Efficacy of exercise on breast cancer outcomes: a systematic review and meta-analysis of preclinical data. Int J Sports Med. 2018; 39: 327-342.

18. Walsh NP, Gleeson M, Shephard RJ, Gleeson M, Woods JA, Bishop NC et al. Position statement. Part one: immune function and exercise. Exerc Immunol Rev. 2011; 17: 6-63.

19. Howard R, Scheiner A, Kanetsky PA, Egan KM. Sociodemographic and lifestyle factors associated with the neutrophil-to-lymphocyte ratio. Ann Epidemiol. 2019; 38: 11-21.

20. Walsh SR, Cook EJ, Goulder F, Justin TA, Keeling NJ. Neutrophil Lymphocyte ratio as a prognostic factor in colorectal cancer. J Surg Oncol. 2005; 91: 181184.

21. Zhang J, Zhang HY, Li J. The elevated NLR, PLR and PLT predict the prognosis of patients with colorectal cancer: a systematic review and metaanalysis. Oncotarget. 2017; 8: 68837-68846.

22. Lee IM, Shiroma EJ, Lobelo F, Puska P, Blair SN, Katzmarzyk PT. Lance Physical Activity Series Working Group. Effect of physical inactivity on major non-communicable diseases worldwide: an analysis of burden of disease and life expectancy. Lancet. 2012; 380: 219-229.

23. Pedersen, L. Idorn $M$, Olofsson $\mathrm{GH}$. Voluntary running suppresses tumo growth through epinephrine- and IL-6-Dependent NK cell mobilization and redistribution. Cell Metab. 2016; 23: 554-562.

24. Templeton AJ, McNamara MG, Seruga B. Prognostic role of neutrophil-tolymphocyte ratio in solid tumors: a systematic review and meta-analysis. J Natl Cancer Inst. 2014; 106: dju124.

25. Ferris RL, Blumenschein G Jr, Fayette J. Nivolumab for Recurrent SquamousCell Carcinoma of the Head and Neck. N Engl J Med. 2016; 375: 1856-1867.

26. Burtness B, Harrington $\mathrm{KJ}$, Greil R. Pembrolizumab alone or with chemotherapy versus cetuximab with chemotherapy for recurrent or metastatic squamous cell carcinoma of the head and neck (KEYNOTE-048): a randomised, open-label, phase 3 study. Lancet. 2019; 394: 1915-1928.

27. Loughney L, West MA, Kemp GJ, Grocott MPW, Jack S. Exercise intervention in people with cancer undergoing neoadjuvant cancer treatment and surgery: a systematic review. Eur J Surg Oncol. 2016; 42: 28-38. 